Case Report

\title{
Home Monitoring of Oxygen Saturation using a Low-cost Wearable Device with Haptic Feedback to Improve Sleep Qual- ity in a Lung Cancer Patient: A Case Report
}

\author{
Walter Lachenmeier ${ }^{1}$, and Dirk W. Lachenmeier ${ }^{2, *}$ \\ 1 Retired from Deutsche Forschungsgemeinschaft (DFG, German Research Foundation), Bonn, Germany; \\ Current address: Kastanienweg 50, 53757 Sankt Augustin, Germany; walter.lachenmeier@web.de \\ 2 Chemisches und Veterinäruntersuchungsamt (CVUA) Karlsruhe, Weissenburger Strasse 3, 76187 Karlsruhe, \\ Germany; lachenmeier@web.de \\ * Correspondence: lachenmeier@web.de; Tel.: +49-721-926-5434
}

\begin{abstract}
The study reports about a case of a lung cancer patient with increasing difficulties in falling asleep and frequent periods of wakefulness. Severe dyspnea related to pneumonitis caused as a side effect of immunotherapy worsened the situation. Eventually, fear of falling asleep developed, including panic attacks and anxiety of choking, which was shown to lead to nights of complete wakefulness. The patient did not only sleep poorly; he did not sleep at all at night for several days, as evidenced by the notes he made during the night. Polygraphy showed no evidence of sleep-disordered breathing, but frequent periods of wakefulness and reduced basal saturation around $90 \%$ during sleep due to lung changes such as extensive functional failure of the left upper lobe with position-dependent shunts. The authors hypothesized that the symptoms described were causally related to a drop in oxygen saturation in the patient's blood. Therefore, they pursued the goal of finding a measurement technique that is as inexpensive as possible and that the patient can operate without outside assistance and great effort. So the patient started using a low-cost wearable device that allows simultaneous measurements of blood oxygen content, pulse rate and movement intensity. It consists of a finger ring with pulse oximetry sensor and a wristband with the control unit containing a vibration motor. The described device reliably warned of disturbances in oxygen concentration in the blood during the night with its vibration alarm. By use of that device during the whole night at home, the events of reduced oxygen saturation and the anxiety symptoms were reduced. Sleep disturbances with sudden awakenings did not occur when using the device. The patient benefited from the security gained in this way and slept much more peacefully, and he could spend nights without waking up again. In conclusion, wearable oximeters with vibration alarm can be recommended for patients' home care in lung cancer patients.
\end{abstract}

Keywords: wearable sensor; pulse oximetry; sleep disturbance; blood oxygenation; haptic feedback; home care; oxygen concentration

\section{Introduction}

Over the last decade, there has been considerable increase in interest and research activity in wearable sensors to measure blood oxygen saturation (SpO2) [1-9]. The principle of pulse oximetry is typically applied, which assesses the level of blood oxygenation by optical light transmission through the blood (for a review of oximetry techniques and their theory, see Nitzan et al. [9]).

Devices are typically constructed as ring-type sensors, which are controlled using cabled or wireless control units, often with the possibility of connecting to a desktop computer or smartphone using USB cable or Bluetooth technology [1,3]. Other approaches suggested the use of $4 \mathrm{G}, \mathrm{WiFi}$, or Zigbee networks to upload data to a server, which could 
be accessed by remote coaches or doctors $[4,8]$. During a validation study against polysomnography in a sleep center, overnight pulse oximetry at home setting was found to provide satisfactory diagnostic performance in detecting severe obstructive sleep apnea [5].

Another innovation was the combination of $\mathrm{SpO}_{2}$ sensors with some form of haptic feedback system, such as an embedded vibration motor [6]. For example, Forra Wakidi et al. suggested a wearable device even in a clinical setting to supervise premature babies. The photodiode sensor combined with a vibrating motor was successfully applied to stimulate the baby's body when apnea occurs, as well as to raise an alarm at the nurse station [2]. The usefulness of such a system to alert patients with obstructive sleep apnea was also described by Brugarolas et al. [7].

The focus of this case report was the application of an easy to use and as simply as possible wearable oximetry device with haptic feedback in a patient with severe sleep disturbances with the objective of resolving his sleep deprivation that is highly detrimental to health.

\section{Case report and methods}

\subsection{Case description}

A 76-year-old man with shortness of breath, productive cough, chest pain, fatigue, and a history of hypertension was diagnosed in November 2020 with non-small cell lung carcinoma (UICC8 classification IIIB). The patient was a smoker with a history of 300 packs per year but had stopped smoking 2 years before diagnosis. Curative radiochemotherapy with carboplatin and paclitaxel was conducted between December 2020 and January 2021, leading to stabilization. The biopsy of a remaining compression in the upper lobe showed no malignancy but only inflammatory tissue due to radiation. Bi-monthly immunotherapy with Durvalumab was initiated in February 2021. Even before the switch to Durvalumab, increasing dyspnea was observed and systemic steroid therapy with 30 $40 \mathrm{mg}$ was started. Progressive dyspnea on exertion was reported during the following period, also insomnia under immunotherapy and panic attacks. The patient reported shortness of breath especially when lying down, so that he used to sleep while sitting at a desk with his head on pillows. In July 2021, a pneumological diagnosis using polygraphy showed no evidence of sleep-disordered breathing, but frequent periods of wakefulness and reduced basal saturation around 90\% during sleep without oxygen due to lung changes. A blood gas analysis under stress showed no respiratory insufficiency, no ventilatory insufficiency, and a balanced $\mathrm{pH}$. Part of the shortness of breath was judged to be pulmonary, certainly explained by the extensive functional failure of the left upper lobe with position-dependent shunts and a narrow connection to the lower lobe. In August 2021, inpatient treatment was necessary after durvalumab infusion because a normocytic normochromic anemia required transfusion for diffuse small intestinal bleeding developed in the patient. The patient was discharged in stable general condition after hospitalization. As the causes of the sleep disturbances were at least partially due to anxiety disorders, including panic attacks and anxiety of choking, the patient started using the wearable device described in section 2.2 in August 2021. However, in September 2021 the severity of dyspnea increased and pneumonitis occurred following resumed immunotherapy leading to further hospitalization. Symptomatic therapy, including oxygen and steroid therapy, stabilized the patient. After discharge, it was decided to abort immunotherapy, which was considered a possible cause of pneumonitis and previous anemia due to unspecific autoimmune responses. Subsequently, the patient considerably improved in being able to sleep through the night. Fatigue, shortness of breath, and coughing were reduced, and the patient is currently stable without the need for oxygen ventilation.

\subsection{Materials and method}

The SleepU device (Wellue Health, Shenzhen Viatom Technology Co., Ltd., Shenzhen, China) was applied. It allows simultaneous measurements of blood oxygen content, 
pulse rate, and movement intensity. It consists of a finger ring with pulse oximetry sensors and a wristband with the control unit containing a vibration motor. The ring is connected to the control unit using a USB cable. The USB port is also used for recharging the device, which is necessary every 2 days. The specified measurement ranges are $70-99 \%$ (oxygen level) and 30-250 bpm (pulse). The measurement interval is 15/min. Advantageous is the intermediate storage of the data, their graphical output on the smartphone, and the possibility of exporting them as a PDF file or CSV file. The ViHealth App for Android V. 2.72.0 (Shenzhen Viatom Technology Co., Ltd., Shenzhen, China) was used for data access and export. For more detailed analysis of the data, Microsoft Excel Version 2019 was applied. The device records \#255 and \#65535 when no reading had been available due to circumstances such as movement or bad signal. These readings were excluded and only valid numbers were used (similar to the approach described by Muratyan et al. [10]). Figure 1 shows the device and application. Sleep experiments of relatively short and long duration (nap and night sleep) were initially performed without oxygen supply. Subsequently, such experiments occurred with oxygen supply from a stationary device Type 525 DS from deVilbiss (Somerset, PA, USA). The SleepU device remained switched on throughout. Figure 1 shows a picture of the device and the application.

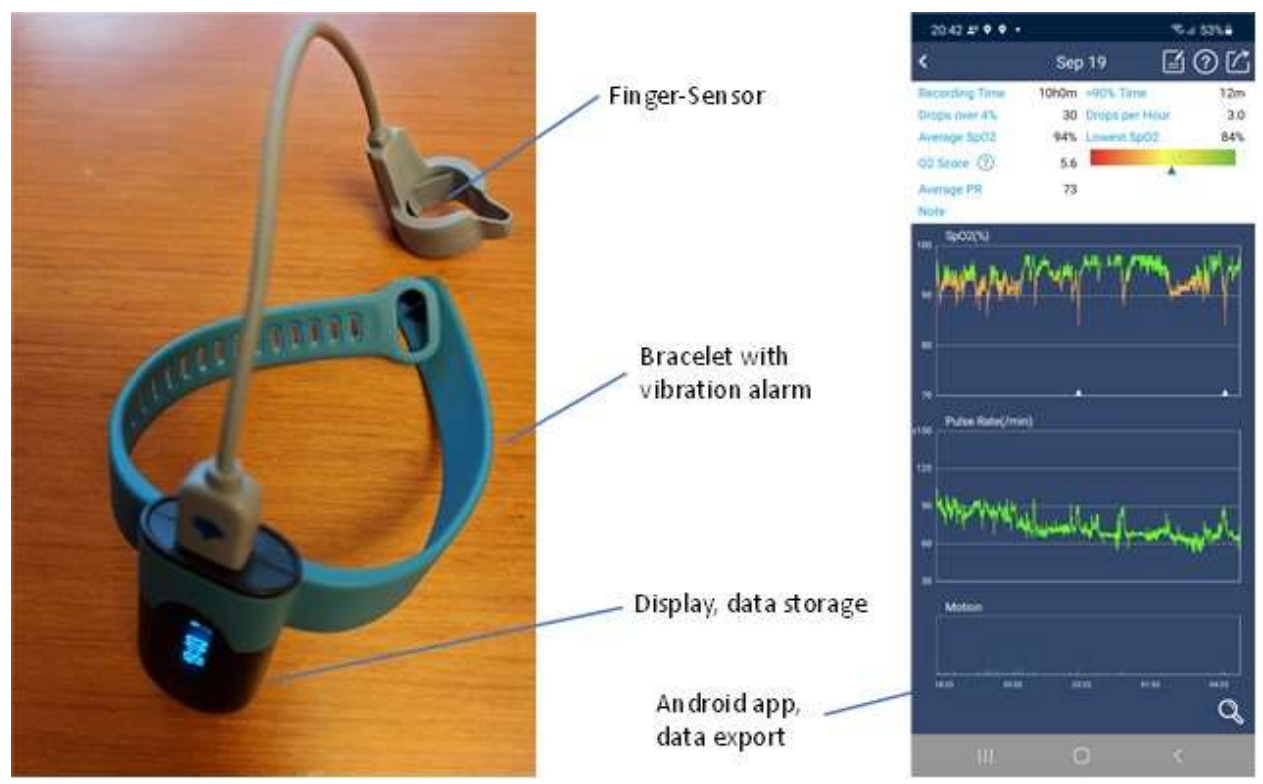

Figure 1. A picture of the device and a screenshot of the data evaluation in the android app.

\section{Results}

Figure 2 shows as a blue curve the course of oxygen saturation in the blood of the patient acutely ill with pneumonitis. In this extreme example, the threshold value of $85 \%$ (blue dashed straight line) was undershot by at least 1\% 26 times between 23:00 and about 4:00. In most cases, the patient awoke during the deficiency phases, which lasted up to 48 seconds. The exact times for falling below the threshold by at least $1 \%$ are shown in Table 1. There was no oxygen supply during the period up to about 4:00 a.m. The oxygen was supplied to the patient only after that. As the blue curve shows, the oxygen saturation then increased significantly within a few seconds and there were no further drops below the threshold value. The pulse per minute of the patient is shown as a green curve. It shows values in the sleep phases just below $80 / \mathrm{min}$ and in the movement phases slightly above $80 / \mathrm{min}$. 


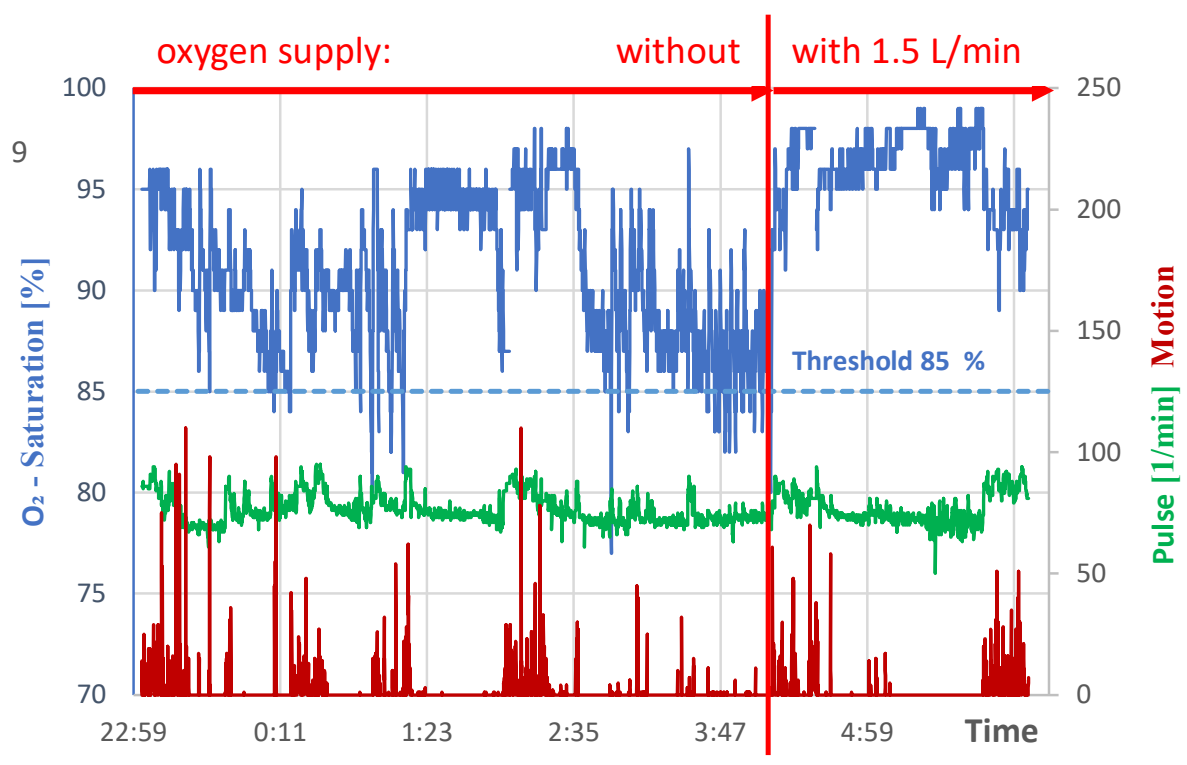

Figure 2. Night sleep of the patient with acute pneumonitis until shortly after 4:00 a.m. without oxygen supply, then with oxygen supply $1.5 \mathrm{~L} / \mathrm{min}$.

The values of the red curve are a measure of the intensity of the movement. The numerical values for the movement (arbitrary units) are not quantified in more detail, but are also not necessary for this investigation. Strong fluctuations in oxygen supply are coupled to phases of intense movement.

For the experiment shown in Figure 2, the duration of the phases of a 1\% fall below the threshold and the corresponding times of start and end are given in Table 1 . The vibration alarm was set to go off at $85 \%$ oxygen saturation. Without the alarm, these undershoots are associated with sudden awakenings and lead to periods of wakefulness if the deficiency is prolonged. The results shown are typical of a number of other experiments that showed very similar results.

Table 1. Reaching and falling below the threshold value ${ }^{1}$ of oxygen concentration in the blood during night sleep according to Figure 2.

\begin{tabular}{cccccc}
\hline Duration Sec. & time from & to & Duration Sec. & time from & to \\
\hline 4 & $0: 08: 31$ & $0: 08: 35$ & 12 & $3: 44: 31$ & $3: 44: 43$ \\
48 & $0: 15: 55$ & $0: 16: 43$ & 20 & $3: 46: 27$ & $3: 46: 47$ \\
8 & $0: 50: 47$ & $0: 50: 55$ & 32 & $3: 49: 27$ & $3: 49: 59$ \\
48 & $0: 55: 55$ & $0: 56: 43$ & 32 & $3: 51: 31$ & $3: 52: 03$ \\
12 & $0: 59: 43$ & $0: 59: 55$ & 32 & $3: 54: 27$ & $3: 54: 59$ \\
44 & $1: 05: 27$ & $1: 06: 11$ & 4 & $3: 55: 03$ & $3: 55: 07$ \\
32 & $1: 11: 31$ & $1: 12: 03$ & 4 & $3: 58: 07$ & $3: 58: 11$ \\
24 & $2: 53: 43$ & $2: 54: 07$ & 32 & $3: 58: 15$ & $3: 58: 47$ \\
12 & $2: 57: 31$ & $2: 57: 43$ & 8 & $4: 01: 55$ & $4: 02: 03$ \\
16 & $3: 01: 59$ & $3: 02: 15$ & 20 & $4: 03: 59$ & $4: 04: 19$ \\
8 & $3: 02: 39$ & $3: 02: 47$ & 16 & $4: 07: 55$ & $4: 08: 11$ \\
20 & $3: 40: 47$ & $3: 41: 07$ & 36 & $4: 11: 31$ & $4: 12: 07$ \\
4 & $3: 41: 35$ & $3: 41: 39$ & 12 & $4: 12: 15$ & $4: 12: 27$ \\
\hline
\end{tabular}

${ }_{1}^{1}$ Triggering of the vibration alarm at $85 \%$. The duration of the undershoot of this threshold value by $1 \%$ is measured. 
Figure 3 shows sleep phases first without, then with oxygen supply to the patient for a sleep of about $1.5 \mathrm{~h}$ duration. At the beginning an awake phase with a fall below the threshold value of longer duration, which is terminated by the patient moving around and breathing more intensively. After the patient falls asleep, further undershoots occur, so that the vibration alarm is triggered and the patient wakes up. The corresponding numerical values are summarized in Table 2.

With oxygen supply starting from approximately 13:00, there is a significantly higher and more balanced profile of the oxygen content in the patient's blood to be observed.

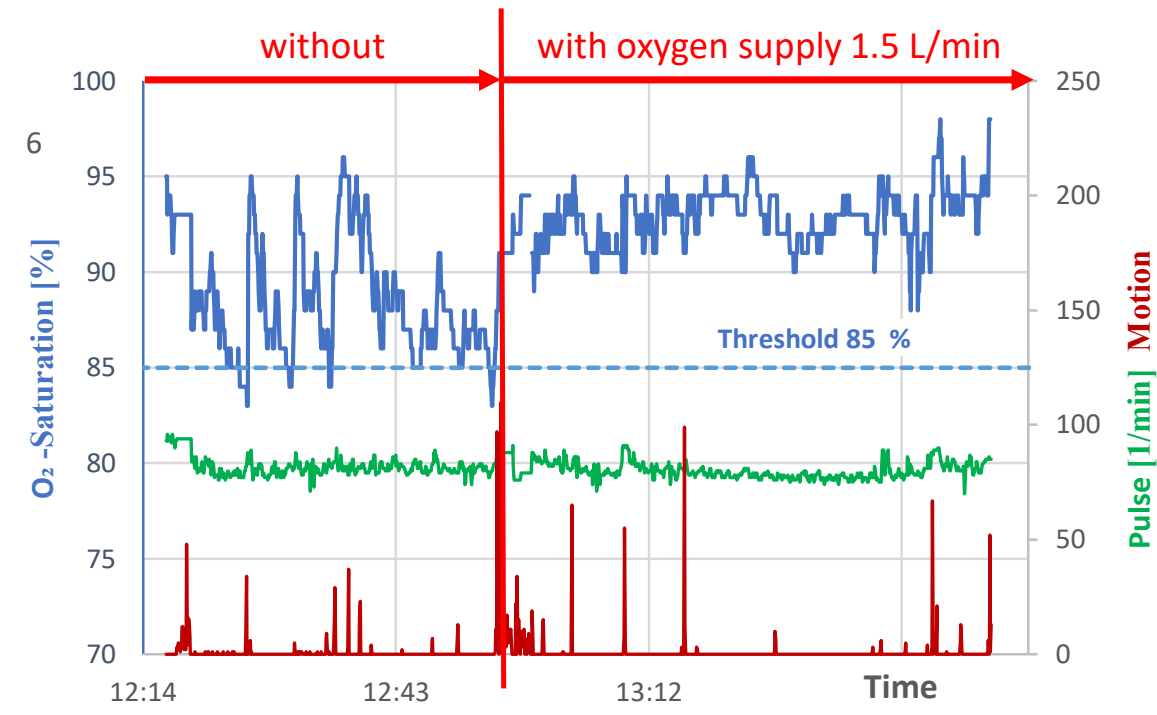

Figure 3. Midday nap of the patient with subsiding pneumonitis until shortly before 13:00 h without oxygen supply, then with oxygen supply $1.5 \mathrm{~L} / \mathrm{min}$.

Table 2. Falling below the threshold value ${ }^{1}$ of the oxygen concentration in the blood by $1 \%$ during the Midday sleep from Figure 3.

\begin{tabular}{ccc}
\hline Duration Sec. & Time from & to \\
\hline 56 & $12: 25: 23$ & $12: 26: 19$ \\
20 & $12: 30: 59$ & $12: 31: 19$ \\
20 & $12: 35: 31$ & $12: 35: 51$ \\
24 & $12: 53: 55$ & $12: 54: 19$ \\
\hline
\end{tabular}

${ }^{1}$ Triggering of the vibration alarm at $85 \%$. The duration of the undershoot of this threshold value by $1 \%$ is measured.

The course of the oxygen concentration in the blood of the almost-recovered patient without oxygen supply during the night sleep is shown in Figure 4 next to pulse course and movement profile. Here, only a single oxygen undersupply occurred (Table 3), whereby the vibration alarm reliably awakened the patient. It lasted 28 seconds and reached a minimum value of $82 \%$. The threshold value of $85 \%$ was reached again later but no value below. According to experience, the detected undersupply occurs within about 10 seconds, starting from a still sufficient oxygen saturation in the range of about $90 \%$. The sensor thus works fast enough to trigger the alarm on time. It is noticeable that the patient's pulse drops to values significantly below $80 /$ minute compared to the case of acute pneumonitis (cf. Figure 2). 


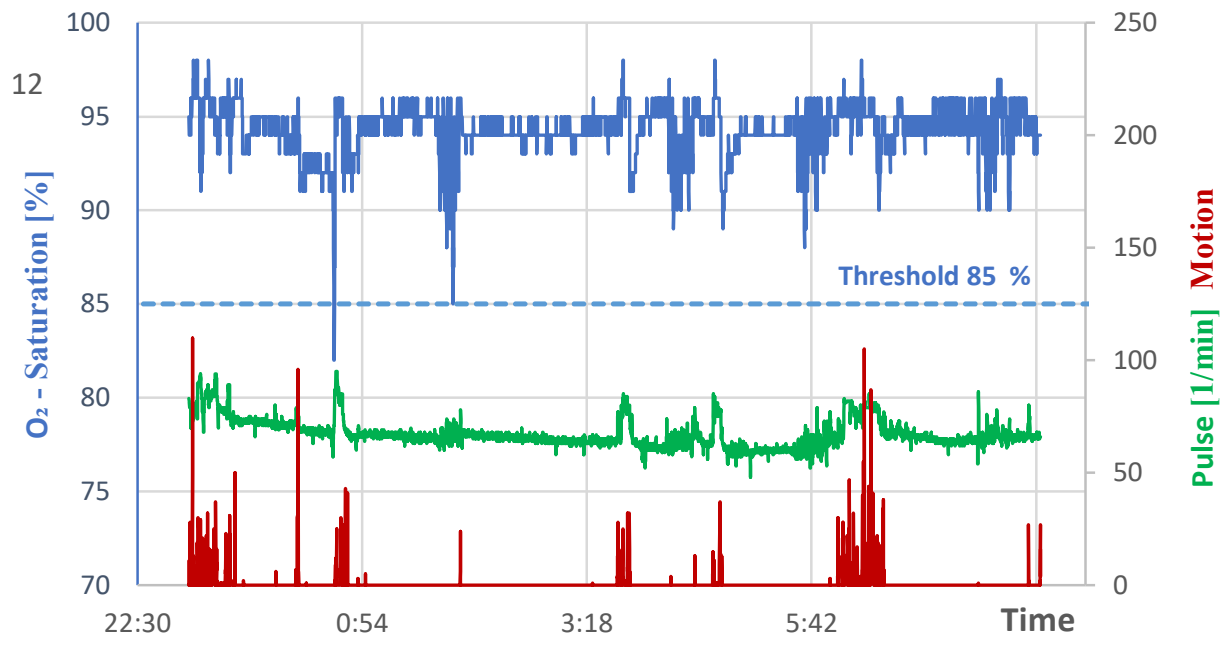

Figure 4. Night sleep of the almost recovered patient without oxygen supply.

Table 3. Falling below the threshold ${ }^{1}$ of the oxygen concentration in the blood during night sleep from Figure 4.

\begin{tabular}{ccc}
\hline Duration Sec. & Time from & to \\
\hline 28 & $0: 36: 33$ & $0: 37: 01$ \\
\hline
\end{tabular}

${ }_{1}^{1}$ Triggering of the vibration alarm at $85 \%$. The duration of the undershoot of this threshold value by $1 \%$ is measured.

\section{Discussion}

The oxygen concentration in the blood is subject to fluctuations in lung cancer patients. The time period of falling below a given threshold value of oxygen concentration was determined. There is no uniform information or agreement in the literature on the level and pathophysiological significance of threshold values for an oxygen shortage, specifically in acutely ill oncologic patients [11-14]. For this work, it was set at $85 \%$ because the patient studied noted a clear relationship to his sleep disturbances in several trials at this value.

He suffered from frequent sudden awakenings from deep sleep or after nightmares. This disturbance progressed to panic attacks over a few weeks. Fear of such attacks later also led to difficulties in falling asleep. It was determined that these problems with sleeping through the night clearly correlate with an undersupply of oxygen in the blood (hypoxemia). On the basis of normal values, the aforementioned threshold value of $85 \%$ is regularly reached within a period of a few seconds. A subsequent undersupply in this range lasts on average between 8 and about 40 seconds. The patient awakens within 4 seconds after the vibration alarm is triggered and can take countermeasures, such as increasing respiratory intensity. Thus, values above $90 \%$ are reached again within a maximum of 40 seconds; only in a few cases, more time was required (Table 1 ). So the evaluated device seems to be well suited to provide patients with sufficient protection and a sense of security during sleep. The advantages of the device include that the data can be transferred easily in the form of PDF reports or CSV files for further evaluations. For this purpose, simple Microsoft Excel evaluations, for example, can be used to selectively view the records in the area of particular interest near the threshold from several thousand rows.

When using the device, the patient will learn from these data which threshold is most appropriate for his individual situation. This means he can determine at which oxygen concentration he starts to move or wakes up and derive from this his individual threshold for the vibration alarm. This suggestion could be a future research topic to implement 
algorithms to suggest an individual threshold value with a few parameters typical for the patient. However, if one wants to do this calculation completely exactly, one would have to assign a caregiver to it, who observes the patient exactly, such as in a sleep lab. Nevertheless, the device covers a lot of what happens in the sleep lab. Patients with severe sleep apnea have up to 100 breathing episodes per night [12]. However, the measurement technique used did not and should not clarify whether these or other possible causes of oxygen deficiency were present.

An advantage is clearly the home use. The patient does not need to go to a sleep lab for one night, which facilitates a tremendous stress, especially for geriatric patients, but the measurements can be done at home. Then the report can be sent to the physician by email who can make the necessary conclusions, such as the need for oxygen supplementation or make suggestions for setting the threshold value for the vibration alarm.

The crucial advantage of the device is the security it gives by the vibration alarm. The patient does not have to be afraid anymore that breathing will stop while being asleep. Especially at the onset of the disease, the patient became afraid of falling asleep and became scared and startled when noticing decreased breathing. This effect has completely disappeared because of the device. Hence the device is especially advantageous for patients, for which sleeping problems may be due to psychological rather than real pathological reasons.

An interesting further application of the same device was published by Muratyan et al. [10]. The data of the device were used to identify the individual user with high accuracy.

Further developments could be to directly couple the device to oxygen supply systems, e.g., using the Bluetooth connection that is already built-in. So instead of the vibration alarm, the oxygen supply could be switched on.

In the future, the applicability and reliability of the device could be improved by including more vital parameters to better guide its decision. For example, respiratory rate and ECG.

A future feature worth investigating would also be the possibility not to set a certain threshold (such as $85 \%$ ), but a percentage deviation from the baseline that would lead to the alarm. For example, Forra Wakidi et al. suggested a 5\% decrease in $\mathrm{SpO}_{2}$ value within $5 \mathrm{~s}$ from the baseline as indicative of apnea [2].

Limitations of the device include the fact that the generalizable applicability is probably limited. Due to the psychological component, not all patients may benefit equally depending on the individual situation. More mentally impaired patients probably will not be able to correctly use the device, which needs to be correctly attached and at least a caregiving person is needed to adjust the app and send the data for analysis.

A downside of the available low-cost consumer devices may be lower accuracy. Regular dropouts were also detected during reading, probably due to lost skin contact with the sensor. A conclusion may therefore be that improvements of the sensors and a more reliable attachment avoiding the slipping off the sensor during the night might be required. However, previous research has shown that finger sensors are typically more reliable in terms of motion artifacts than other types of sensors [1,2].

The starting point of this evaluation was the aim of improving sleep behavior, including time to fall asleep and length of sleep. The results show that the prevailing sleep disorder of the patient could be significantly reduced with the device. Over the course of time, this might also increase survival, which was strongly correlated with $\mathrm{SpO}_{2}$ levels in lung cancer patients [11].

\section{Conclusions}

Sleep disturbances can be precursors to serious health problems, as can problems with night terrors or sleep apnea. The study investigated whether the simple measurement of the time course of the concentration of oxygen in the blood is suitable for detecting sudden respiratory disturbances sufficiently quickly for a prompt and effective warning 
by a vibration alarm. Indeed, the described device reliably warned of disturbances in oxygen concentration in the blood using a vibration alarm. Sleep disturbances with sudden awakening did not occur when the device was used. The patient benefited from the security gained in this way and slept much more peacefully. This study also substantiates the hypothesis that oxygen deficiency in the blood had been a major cause of insomnia in the patient.

Supplementary Materials: The following is available online at https://www.preprints.org/manuscript/202111.0331/v1/download/supplementary, Table S1: Raw data in Microsoft Excel format.

Author Contributions: Conceptualization, W.L.; methodology, W.L.; formal analysis, W.L.; investigation, W.L.; data curation, W.L.; writing - original draft preparation, W.L.; writing - review and editing, D.W.L.; visualization, W.L. All authors have read and agreed to the published version of the manuscript.

Funding: This research received no external funding.

Institutional Review Board Statement: The study was carried out according to the guidelines of the Declaration of Helsinki. Ethical review and approval were waived for this study because this study did not involve more than minimal risk because it did not involve a prospective evaluation and only involved a non-invasive procedure.

Informed Consent Statement: Written informed consent has been obtained from the patient to publish this article.

Data Availability Statement: All data generated or analyzed during this study are included in this published article (and its supplementary materials).

Acknowledgments: Reference in this article to any specific commercial products, process, service, manufacturer, company, or trademark does not constitute its endorsement or recommendation by the authors or any affiliated institute.

Conflicts of Interest: The authors declare no conflict of interest. The authors specifically stress that the research was not funded or supported by the manufacturer of the evaluated device. The authors selected the device following market research on the internet, because of its features, consumer rating, comparably low price and immediate availability, and paid for it with own funds.

\section{References}

1. Sokwoo, R.; Boo-Ho, Y.; Kuowei, C.; Asada, H.H. The ring sensor: a new ambulatory wearable sensor for twenty-four hour patient monitoring. In Proceedings of the 20th Annual International Conference of the IEEE Engineering in Medicine and Biology Society. Vol. 20 Biomedical Engineering Towards the Year 2000 and Beyond (Cat.No.98CH36286), IEEE: Manhattan, New York, U.S., 1998; pp 19061909. doi:10.1109/IEMBS.1998.746970

2. Forra Wakidi, L.; Hari Wisana, I.D.; Miftahul Maghfiroh, A.; Sharma, V.K. Apnea monitor using pulse oxymetry with tactile stimulation to reduce respiration failure. J. Electronic. Electromed. Eng. Med. Inform. 2021, 3, 79-84. doi:10.35882/jeeemi.v3i2.3

3. Chen, Q.; Tang, L. A wearable blood oxygen saturation monitoring system based on bluetooth low energy technology. Comput. Commun. 2020, 160, 101-110. doi:10.1016/j.comcom.2020.05.041

4. Fu, Y.; Liu, J. System design for wearable blood oxygen saturation and pulse measurement device. Procedia Manuf. 2015, 3, 1187-1194. doi:10.1016/j.promfg.2015.07.197

5. Hang, L.W.; Wang, H.L.; Chen, J.H.; Hsu, J.C.; Lin, H.H.; Chung, W.S.; Chen, Y.F. Validation of overnight oximetry to diagnose patients with moderate to severe obstructive sleep apnea. BMC Pulm. Med. 2015, 15, 24. doi:10.1186/s12890-015-0017-z

6. Sanfilippo, F.; Pettersen, K.Y. A sensor fusion wearable health-monitoring system with haptic feedback. In 11th International Conference on Innovations in Information Technology (IIT), IEEE: Manhattan, New York, U.S., 2015; pp 262-266. doi:10.1109/INNOVATIONS.2015.7381551 
7. Brugarolas, R.; Valero-Sarmiento, J.M.; Brna, A. Wearable SpO2 and sleep posture monitoring system for obstructive sleep apnea patients. In IEEE Virtual Conference on Applications of Commercial Sensors (VCACS), IEEE: Manhattan, New York, U.S., 2015; pp 1-6. doi:10.1109/VCACS.2015.7439613

8. Adiputra, R.R.; Hadiyoso, S.; Hariyani, Y.S. Internet of things: Low cost and wearable SpO2 device for health monitoring. Int. J. Electr. Comput. Eng. 2018, 8, 939. doi:10.11591/ijece.v8i2.pp939-945

9. Nitzan, M.; Nitzan, I.; Arieli, Y. The various oximetric techniques used for the evaluation of blood oxygenation. Sensors (Basel, Switzerland) 2020, 20, 4844. doi:10.3390/s20174844

10. Muratyan, A.; Cheung, W.; Dibbo, S.V.; Vhaduri, S. Opportunistic multi-modal user authentication for health-tracking IoT wearables. arXiv 2021, 2109.13705.

11. Martins, S.J.; Ho, N.; Cavamura, S.O.; Harada, C.M.; Yamamoto, C.A.; Takagaki, T.Y. Lung cancer symptoms and pulse oximetry in the prognostic assessment of patients with lung cancer. BMC Cancer 2005, 5, 72. doi:10.1186/1471-2407-5-72

12. Zander, R. Pathophysiologie des arteriellen O2-Status; https://www.physioklin.de/physiooxy/pathophysiologie-des-arterielleno2-status.html: [accessed on 2021-10-12], 2021

13. Siemieniuk, R.A.C.; Chu, D.K.; Kim, L.H.-Y.; Güell-Rous, M.R.; Alhazzani, W.; Soccal, P.M.; Karanicolas, P.J.; Farhoumand, P.D.; Siemieniuk, J.L.K.; Satia, I.; Irusen, E.M.; Refaat, M.M.; Mikita, J.S.; Smith, M.; Cohen, D.N.; Vandvik, P.O.; Agoritsas, T.; Lytvyn, L.; Guyatt, G.H. Oxygen therapy for acutely ill medical patients: a clinical practice guideline. BMJ 2018, 363 , k4169. doi:10.1136/bmj.k4169

14. Grensemann, J.; Wachs, C.; Kluge, S. Sauerstofftherapie in der Notfall- und Intensivmedizin. Dtsch. Med. Wochenschr. 2021, 146, 108-120. doi:10.1055/a-0948-8363 